\title{
Analysis of discontinuous Galerkin methods using mesh-dependent norms and applications to problems with rough data
}

\author{
Emmanuil H. Georgoulis ${ }^{1,2}$. Tristan $\operatorname{Pryer}^{3}$ (D)
}

Received: 29 March 2017 / Accepted: 22 September 2017 / Published online: 1 November 2017 C The Author(s) 2017. This article is an open access publication

\begin{abstract}
We prove the inf-sup stability of a discontinuous Galerkin scheme for second order elliptic operators in (unbalanced) mesh-dependent norms for quasi-uniform meshes for all spatial dimensions. This results in a priori error bounds in these norms. As an application we examine some problems with rough source term where the solution can not be characterised as a weak solution and show quasi-optimal error control.
\end{abstract}

Keywords Discontinuous Galerkin methods · Inf-sup condition · Rough problem data

Mathematics Subject Classification 65 N12 $\cdot 65$ N30

\section{Introduction}

Discontinuous Galerkin ( $\mathrm{dG}$ ) methods are a popular family of non-conforming finite element-type approximation schemes for partial differential equations (PDEs) involving discontinuous approximation spaces. In the context of elliptic problems their inception can be traced back to the 1970s [5,21]; see also [1] for an accessible overview

Tristan Pryer

T.Pryer@reading.ac.uk

Emmanuil H. Georgoulis

Emmanuil.Georgoulis@le.ac.uk

1 Department of Mathematics, University of Leicester, University Road, Leicester LE1 7RH, UK

2 Department of Mathematics, School of Applied Mathematical and Physical Sciences, National Technical University of Athens, 15780 Zografou, Greece

3 Department of Mathematics and Statistics, University of Reading, Whiteknights, PO Box 220, Reading RG6 6AX, UK 
and history of these methods for second order problems. For higher order problems, for example the (nonlinear) biharmonic problem, $\mathrm{dG}$ methods are a useful alternative to using $\mathrm{C}^{1}$-conforming elements whose derivation and implementation can become very complicated $[5,13,23]$.

Inf-sup conditions form one part of the Banach-Nečas-Babuška condition which guarantees the well-posedness of a given variational problem. In this note, we shall describe an analytical framework to examine the stability of $\mathrm{dG}$ approximations for $\mathrm{L}_{2}$ and $\mathrm{H}^{2}$-like mesh-dependent norms. This is in keeping with the spirit of [3,4], where for continuous finite element methods the authors prove equivalent results for second and fourth order problems respectively. The present approach, however, is quite different and results in inf-sup stability for both $\mathrm{L}_{2}$ - and $\mathrm{H}^{2}$-like mesh-dependent norms under the assumption that the underlying mesh is quasi-uniform.

The analysis presented utilises a new $\mathrm{H}^{2}$-conforming reconstruction operator, based on Hsieh-Clough-Tocher-type $\mathrm{C}^{1}$ reconstructions. Such reconstructions, based on nodal averaging, are used for the proof of a posteriori bounds for non-conforming methods for elliptic [7, 13, 18,24] and hyperbolic problems [12,16]. The new reconstruction operators presented below enjoys certain orthogonality properties; in particular, they are adjoint orthogonal to the underlying Hsieh-Clough-Tocher space and maintain the same stability bounds as the $\mathrm{H}^{2}$-conforming reconstruction from [13].

The argument is quite general and allows the derivation of inf-sup stability results whenever the numerical scheme has a well posed discrete adjoint (dual) problem over an appropriately constructed non-conforming finite element space. This is contrary to the Aubin-Nitsche $\mathrm{L}_{2}$ duality argument whereby it is the underlying partial differential operator itself that requires the well posedness of the adjoint continuous problem.

The use of these recovery operators is not limited to an a posteriori setting, indeed, they have been used to quantify inconsistencies appearing in standard interior penalty methods when the exact solution is not $\mathrm{H}^{2}(\Omega)$ [17]. This allows for quasi optimal a priori bounds for elliptic problems under minimal regularity up to data oscillation. Fundamentally the assumption in this analysis is that the singularity arises from the geometry of the domain rather than through the problem data itself. Our analysis allows us to show quasi-optimal $\mathrm{L}_{2}$ convergence to problems that have rough problem data. To showcase the result we study the convergence of a method posed for an elliptic problem whose source term is not $\mathrm{H}^{-1}$ in both 1 and 2 spatial dimensions. In this case the AubinNitsche, and indeed the standard treatment of Galerkin methods, are not applicable.

The note is set out as follows: In Sect. 2 we introduce the problem and present the analysis cumulating in inf-sup stability for problems with smooth data. In Sect. 3 we examine a particular problem with rough data and prove quasi-optimal convergence in this case. In addition we give some numerical validation of the method.

\section{Problem set up and discretisation}

To highlight the main steps of the present developments in this area, we consider the Poisson problem with homogeneous Dirichlet boundary conditions. Let $\Omega \subset \mathbb{R}^{d}$ be an open convex domain and consider the problem: given $f \in \mathrm{L}_{2}(\Omega)$ find $u \in$ $\mathrm{H}^{2}(\Omega) \cap \mathrm{H}_{0}^{1}(\Omega)$, such that 


$$
\int_{\Omega} \nabla u \cdot \nabla v \mathrm{~d} x=\int_{\Omega} f v \mathrm{~d} x \quad \forall v \in \mathrm{H}_{0}^{1}(\Omega) .
$$

We consider $\mathscr{T}$ to be a conforming triangulation of $\Omega$, namely, $\mathscr{T}$ is a finite family of sets such that

(1) $K \in \mathscr{T}$ implies $K$ is an open simplex (segment for $d=1$, triangle for $d=2$, tetrahedron for $d=3$ ),

(2) for any $K, J \in \mathscr{T}$ we have that $\bar{K} \cap \bar{J}$ is a full lower-dimensional simplex (i.e., it is either $\varnothing$, a vertex, an edge, a face, or the whole of $\bar{K}$ and $\bar{J}$ ) of both $\bar{K}$ and $\bar{J}$ and

(3) $\bigcup_{K \in \mathscr{T}} \bar{K}=\bar{\Omega}$.

The shape regularity constant of $\mathscr{T}$ is defined as the number

$$
\mu(\mathscr{T}):=\inf _{K \in \mathscr{T}} \frac{\rho_{K}}{h_{K}},
$$

where $\rho_{K}$ is the radius of the largest ball contained inside $K$ and $h_{K}$ is the diameter of $K$. An indexed family of triangulations $\left\{\mathscr{T}^{n}\right\}_{n}$ is called shape regular if

$$
\mu:=\inf _{n} \mu\left(\mathscr{T}^{n}\right)>0 .
$$

Further, we define $h: \Omega \rightarrow \mathbb{R}$ to be the piecewise constant meshsize function of $\mathscr{T}$ given by

$$
h(\mathbf{x}):=\max _{\bar{K} \ni \mathbf{x}} h_{K} .
$$

In addition if

$$
\frac{\max _{K \in \mathscr{T}} h_{K}}{\min _{K \in \mathscr{T}} h_{K}} \leq C_{q u},
$$

we call $\mathscr{T}$ quasiuniform. If an entire indexed family of triangulations satisfy (4), we call it a quasiuniform family. In what follows we shall assume that all triangulations are shape-regular and quasiuniform.

We let $\mathscr{E}$ be the skeleton (set of common interfaces) of the triangulation $\mathscr{T}$ and say $e \in \mathscr{E}$ if $e$ is on the interior of $\Omega$ and $e \in \partial \Omega$ if $e$ lies on the boundary $\partial \Omega$ and set $h_{e}$ to be the diameter of $e$. We also define the "broken" gradient $\nabla_{h}$, Laplacian $\Delta_{h}$ and Hessian $\mathrm{D}_{h}^{2}$ to be defined element-wise by $\left.\nabla_{h} w\right|_{K}=\nabla w,\left.\Delta_{h} w\right|_{K}=\Delta w$, $\left.\mathrm{D}_{h}^{2} w\right|_{K}=\mathrm{D}^{2} w$ for all $K \in \mathscr{T}$, respectively, for respectively smooth functions on the interior of $K$,

We let $\mathbb{P}^{k}(\mathscr{T})$ denote the space of piecewise polynomials of degree $k$ over the triangulation $\mathscr{T}$, and introduce the finite element space $\mathbb{V}:=\mathbb{P}^{k}(\mathscr{T})$ to be the usual space of discontinuous piecewise polynomial functions of degree $k$. We define average operators for arbitrary scalar functions $v$ and vectors $\mathbf{v}$ over an edge $e$ shared by elements $K_{1}$ and $K_{2}$ as $\left\{\{v\}=\frac{1}{2}\left(\left.v\right|_{K_{1}}+\left.v\right|_{K_{2}}\right)\right.$, $\left\{\{\mathbf{v}\}=\frac{1}{2}\left(\left.\mathbf{v}\right|_{K_{1}}+\left.\mathbf{v}\right|_{K_{2}}\right)\right.$ and jump operators as $\llbracket v \rrbracket=\left.v\right|_{K_{1}} \boldsymbol{n}_{K_{1}}+\left.v\right|_{K_{2}} \boldsymbol{n}_{K_{2}}, \llbracket \mathbf{v} \rrbracket=\left.\mathbf{v}\right|_{K_{1}} \cdot \boldsymbol{n}_{K_{1}}+\left.\mathbf{v}\right|_{K_{2}} \cdot \boldsymbol{n}_{K_{2}}$. Note that 
on the boundary of the domain $\partial \Omega$ the jump and average operators are defined as $\left\{\left.\{v\}\right|_{\partial \Omega}:=v,\left\{\left.\{\boldsymbol{v}\}\right|_{\partial \Omega}:=\boldsymbol{v},\left.\llbracket v \rrbracket\right|_{\partial \Omega}:=v \boldsymbol{n},\left.\llbracket \boldsymbol{v} \rrbracket\right|_{\partial \Omega}:=\boldsymbol{v} \cdot \boldsymbol{n}\right.\right.$,

Definition 2.1 (Mesh dependent norms) We introduce the mesh dependent $\mathrm{L}_{2}-, \mathrm{H}^{1}$ and $\mathrm{H}^{2}$-norms to be

$$
\begin{aligned}
\left\|w_{h}\right\|_{0, h}^{2} & :=\left\|w_{h}\right\|_{\mathrm{L}_{2}(\Omega)}^{2}+\left\|h^{3 / 2}\left\{\nabla w_{h}\right\}\right\|_{\mathrm{L}_{2}(\mathscr{E} \cup \partial \Omega)}^{2}+\left\|h^{1 / 2} \llbracket w_{h} \rrbracket\right\|_{\mathrm{L}_{2}(\mathscr{E} \cup \partial \Omega)}^{2} \\
\left\|w_{h}\right\|_{1, h}^{2} & :=\left\|\nabla_{h} w_{h}\right\|_{\mathrm{L}_{2}(\Omega)}^{2}+\left\|h^{-1 / 2} \llbracket w_{h} \rrbracket\right\|_{\mathrm{L}_{2}(\mathscr{E} \cup \partial \Omega)}^{2} \\
\left\|w_{h}\right\|_{2, h}^{2} & :=\left\|\Delta_{h} w_{h}\right\|_{\mathrm{L}_{2}(\Omega)}^{2}+\left\|h^{-1 / 2} \llbracket \nabla w_{h} \rrbracket\right\|_{\mathrm{L}_{2}(\mathscr{E})}^{2}+\left\|h^{-3 / 2} \llbracket w_{h} \rrbracket\right\|_{\mathrm{L}_{2}(\mathscr{E} \cup \partial \Omega)}^{2} .
\end{aligned}
$$

Note for $w_{h} \in \mathbb{V}$ in view of scaling each mesh dependent norm is equaivalent to the continuous counterpart, that is $\left\|w_{h}\right\|_{0, h} \sim\left\|w_{h}\right\|_{\mathrm{L}_{2}(\Omega)}$ for example.

Consider the interior penalty (IP) discretisation of (1), to find $u_{h} \in \mathbb{V}$ such that

$$
\mathscr{A}_{h}\left(u_{h}, v_{h}\right)=\left\langle f, v_{h}\right\rangle \quad \forall v_{h} \in \mathbb{V},
$$

where

$$
\begin{aligned}
\mathscr{A}_{h}\left(u_{h}, v_{h}\right)= & \int_{\Omega} \nabla_{h} u_{h} \cdot \nabla_{h} v_{h} \mathrm{~d} x-\int_{\mathscr{E} \cup \partial \Omega} \llbracket u_{h} \rrbracket \cdot\left\{\left\{\nabla v_{h} \rrbracket+\llbracket v_{h} \rrbracket \cdot\left\{\nabla_{h} \rrbracket\right] \mathrm{d} s\right.\right. \\
& +\int_{\mathscr{E} \cup \partial \Omega} \frac{\sigma_{0}}{h} \llbracket u_{h} \rrbracket \cdot \llbracket v_{h} \rrbracket \mathrm{d} s+\int_{\mathscr{E}} \sigma_{1} h \llbracket \nabla u_{h} \rrbracket \cdot \llbracket \nabla v_{h} \rrbracket \mathrm{d} s,
\end{aligned}
$$

where $\sigma_{0}>0, \sigma_{1} \geq 0$ represent penalty parameters. Note that a standard choice is to take $\sigma_{1}=0$. The choice $\sigma_{1} \neq 0$ results in a class of stabilised $\mathrm{dG}$ methods [8].

Proposition 2.2 (Continuity and coercivity of $\mathscr{A}_{h}(\cdot, \cdot)\left[1,11\right.$, c.f.]) For $\sigma_{1} \geq 0$ and $\sigma_{0}$ large enough and any $u_{h}, v_{h} \in \mathbb{V}$ the bilinear form $\mathscr{A}_{h}(\cdot, \cdot)$ satisfies

$$
\begin{aligned}
& \mathscr{A}_{h}\left(u_{h}, u_{h}\right) \geq C\left\|u_{h}\right\|_{1, h}^{2} \\
& \mathscr{A}_{h}\left(u_{h}, v_{h}\right) \leq C\left\|u_{h}\right\|_{1, h}\left\|v_{h}\right\|_{1, h} .
\end{aligned}
$$

Lax-Milgram Theorem guarantees a unique solution to the problem (7). Also, since $u \in \mathrm{H}^{2}(\Omega)$, the bilinearform is consistent, hence, Strang's Lemma yields quasioptimal convergence of the method in the $\|\cdot\|_{1, h}$ norm:

$$
\left\|u-u_{h}\right\|_{1, h} \leq C \inf _{w_{h} \in \mathbb{V}}\left\|u-w_{h}\right\|_{1, h} .
$$

Conforming reconstruction operators The key tool in the proof of the inf-sup condition is the notion of reconstruction operators. It is commonplace in the a posteriori analysis of nonconforming schemes to make use of such operators. A simple, quite general methodology for the construction of reconstruction operators is to use an averaging interpolation operator into an $\mathrm{H}^{2}$-conforming finite element space. For example a $\mathrm{C}^{1}$ Hsieh-Clough-Tocher (HCT) macro-element conforming space for $\mathrm{H}^{2}$ conformity $[6,13,25$, c.f.]. Another option is the use of Argyris-type reconstructions [6]. 


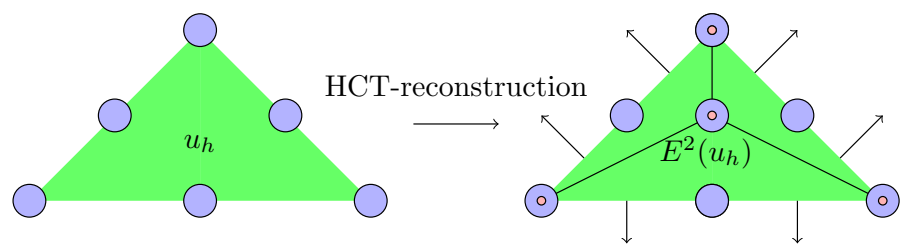

Fig. 1 The $\mathbb{P}^{4}$ Hsieh-Clough-Tocher-type macro-element, as a $\mathrm{H}^{2}(\Omega)$-conforming reconstruction to the quadratic Lagrange element. Here the large, blue circles represent degrees of freedom associated to a function evaluation, the small, red circles represent those associated to full derivative evaluation and the arrows a normal derivative evaluation

Example 2.3 (Construction of the HCT(4) space for $d=2$ ) Since the HCT spaces are an integral part of our analysis, we will illustrate the construction of the HCT(4) space for $d=2$, over triangles, noting that for $d=1$, we can simply consider families of cubic splines, while for $d=3$ corresponding constructions are possible [19, Ch. 18]. Consider a triangle $K$ that is partitioned in 3 subtriangles, $\left\{K_{i}\right\}_{i=1}^{3}$ by connecting each of the vertices to the barycentre as illustrated in Fig. 1. We then take

$$
\operatorname{HCT}(4):=\left\{\phi \in \mathrm{C}^{1}(K):\left.u\right|_{K_{i}} \in \mathbb{P}^{4}\left(K_{i}\right) \text { for } i=1,2,3\right\} .
$$

The dimension of $\mathbb{P}^{4}\left(K_{i}\right)$ is 15 . It can then be verified that, upon enforcing continuity of functions and their derivatives across the subtriangulation edges for the degrees of freedom depicted in Fig. 1, the dimension of the HCT(4) space is 21 and those degrees of freedom and unisolvent.

For the general construction of the macro-element $\operatorname{HCT}(r)$ space for simplicial and box-type elements, we refer to $[10,19,22]$.

Definition 2.4 $\left(\mathrm{H}^{2}(\Omega)\right.$-reconstructions) An example of $\mathrm{H}^{2}(\Omega)$ reconstruction operator $E^{2}\left(u_{h}\right)$ of $k$-th order Lagrange elements is defined as follows. Let $\mathbf{x}$ be a degree of freedom of the $H^{2}$-conforming space $\mathrm{HCT}(k+2)$ consisting of HCT-type macroelements of degree $k+2$, and let $\bar{K}_{\mathbf{x}}$ be the set of all elements sharing the degree of freedom $\mathbf{x}$. Then, the reconstruction at that specific degree of freedom is given by

$$
E^{2}\left(u_{h}\right)(\mathbf{x})=\left.\frac{1}{\operatorname{card}\left(\widehat{K_{\mathbf{x}}}\right)} \sum_{K \in \widehat{K}_{\mathbf{x}}} u_{h}\right|_{K}(\mathbf{x}) \text {. }
$$

For the case $k=2$, the associated degrees of freedom are illustrated in Fig. 1. Notice that the degrees of freedom of the reconstruction are a superset of those of the original finite element. This is due to the lack of existence of a conforming $\mathrm{H}^{2}(\Omega)$ subspace in $\mathbb{V}$ for low $k$; for instance, the existence of an $\mathrm{H}^{2}(\Omega)$-conforming space requires $k \geq 5$ in two dimensions (Argyris space). Corresponding reconstructions for higher polynomial degrees have been considered in $[6,13]$, for instance.

Even though the $\operatorname{HCT}(k+2)$ space contains functions that are not polynomial, it does include $\mathbb{P}^{k+2}(K)$ and hence the $\operatorname{HCT}(k+2)$ interpolant preserves $\mathbb{P}^{k+2}(K)$ 
functions, hence by Bramble-Hilbert the HCT $(k+2)$ space has quasi-optimal approximability.

We refer also to the discussion in [13], regarding reconstructions of box-type $k$-th order Lagrange elements into $H C T(k+2)$.

Lemma 2.5 (Reconstruction bounds [13, Lem 3.1]) For $d=1$, 2, the HCT $(k+2)$ reconstruction operator $E^{2}: \mathbb{V} \rightarrow \mathrm{H}^{2}(\Omega)$ satisifies the following bound for all $u_{h} \in \mathbb{V}$ :

$$
\left\|E^{2}\left(u_{h}\right)-u_{h}\right\|_{1, h}^{2} \leq C\left(\left\|h^{1 / 2} \llbracket \nabla u_{h} \rrbracket\right\|_{\mathrm{L}_{2}(\mathscr{E})}^{2}+\left\|h^{-1 / 2} \llbracket u_{h} \rrbracket\right\|_{\mathrm{L}_{2}(\mathscr{E})}^{2}\right)
$$

with the constant $C>0$ independent of $u_{h}$ and of $h$.

Remark 2.6 Lemma 2.5 is proven in [13] for $d=2$. For $d=1$, we can recover into cubic or quartic splines and the proof is completely analogous. The proof for $d=3$ using one of the trivariate $C^{1}$-elements with nodal and normal derivative degrees of freedom presented in [19] is conjectured to follow along the same lines to the proof of [13, Lem 3.1].

Using this $\operatorname{HCT}(k+2)$-reconstruction, we can construct a further $\operatorname{HCT}(k+2)$ reconstruction admitting the same bounds, but also satisfying an adjoint orthogonality property.

Definition 2.7 (HCT $(k+2)$-Ritz reconstruction) We define the Hsieh-CloughTocher $\mathrm{H}^{2}(\Omega)$-conforming Ritz reconstruction operator $E^{\mathscr{R}}: \mathbb{V} \rightarrow \mathrm{HCT}(k+2)$ such that

$$
\int_{\Omega} \nabla E^{\mathscr{R}}\left(u_{h}\right) \cdot \nabla \tilde{v} \mathrm{~d} x=\int_{\Omega} \nabla_{h} u_{h} \cdot \nabla \tilde{v} \mathrm{~d} x-\int_{\mathscr{E} \cup \partial \Omega} \llbracket u_{h} \rrbracket \cdot \nabla \widetilde{v} \mathrm{~d} s \quad \forall \widetilde{v} \in \operatorname{HCT}(k+2) .
$$

Lemma 2.8 (Properties of $E^{\mathscr{R}}$ ) The HCT $(k+2)$-Ritz reconstruction is well-defined and satisfies the orthogonality condition:

$$
\int_{\Omega}\left(u_{h}-E^{\mathscr{R}}\left(u_{h}\right)\right) \Delta \widetilde{v}=0 \quad \forall \tilde{v} \in \operatorname{HCT}(k+2) .
$$

In addition, for $\alpha=0,1,2$, we have

$$
\sum_{K \in \mathscr{T}}\left|E^{\mathscr{R}}\left(u_{h}\right)-u_{h}\right|_{\mathrm{H}^{\alpha}(K)}^{2} \leq C\left(\left\|h^{3 / 2-\alpha} \llbracket \nabla u_{h} \rrbracket\right\|_{\mathrm{L}_{2}(\mathscr{E})}^{2}+\left\|h^{1 / 2-\alpha} \llbracket u_{h} \rrbracket\right\|_{\mathrm{L}_{2}(\mathscr{E})}^{2}\right),
$$

for $C>0$ constants, independent of $u_{h}$ and of $h$.

Proof Fixing $u_{h} \in \mathbb{V}, E^{\mathscr{R}}\left(u_{h}\right)$ is well-defined. Indeed, setting $\widetilde{v}=E^{\mathscr{R}}\left(u_{h}\right)$ in (14), along with a standard inverse estimate, we deduce

$$
\left\|\nabla E^{\mathscr{R}}\left(u_{h}\right)\right\|_{\mathrm{L}_{2}(\Omega)} \leq C\left\|u_{h}\right\|_{1, h},
$$


for $C>0$ independent of $u_{h}$. The orthogonality condition follows from integrating both sides of (14) by parts.

To see (16) we note that

$$
\begin{aligned}
\left\|E^{\mathscr{R}}\left(u_{h}\right)-u_{h}\right\|_{1, h}^{2} & \leq C \mathscr{A}_{h}\left(E^{\mathscr{R}}\left(u_{h}\right)-u_{h}, E^{\mathscr{R}}\left(u_{h}\right)-u_{h}\right) \\
& =C \mathscr{A}_{h}\left(E^{\mathscr{R}}\left(u_{h}\right)-u_{h}, E^{2}\left(u_{h}\right)-u_{h}\right) \\
& \leq C\left\|E^{\mathscr{R}}\left(u_{h}\right)-u_{h}\right\|_{1, h}\left\|E^{2}\left(u_{h}\right)-u_{h}\right\|_{1, h} .
\end{aligned}
$$

Notice that in order to invoke coercivity of $\mathscr{A}_{h}$ over $W(h)$ we must choose $\sigma_{0}$ larger than if we merely required coercivity over $\mathbb{V}$ since, as already mentioned in Example 2.4, $W(h)$ contains piecewise polynomials two degrees higher than $\mathbb{V}$. Using the properties of $E^{2}\left(u_{h}\right)$ from Lemma 2.5 shows the claim for $\alpha=1$. The result for $\alpha=2$ follows by an inverse inequality.

For $\alpha=0$ we use a duality argument. Take $z \in \mathrm{H}^{2}(\Omega) \cap \mathrm{H}_{0}^{1}(\Omega)$ as the solution of the dual problem

$$
-\Delta z=E^{\mathscr{R}}\left(u_{h}\right)-u_{h}
$$

then

$$
\begin{aligned}
\left\|E^{\mathscr{R}}\left(u_{h}\right)-u_{h}\right\|_{\mathrm{L}_{2}(\Omega)}^{2} & =\int_{\Omega}-\Delta z\left(E^{\mathscr{R}}\left(u_{h}\right)-u_{h}\right) \mathrm{d} x \\
& =\int_{\Omega}-(\Delta z-\Delta \widetilde{z})\left(E^{\mathscr{R}}\left(u_{h}\right)-u_{h}\right) \mathrm{d} s \quad \forall \widetilde{z} \in \operatorname{HCT}(k+2),
\end{aligned}
$$

in view of the orthogonality property (15). Integrating by parts we see

$$
\begin{aligned}
\left\|E^{\mathscr{R}}\left(u_{h}\right)-u_{h}\right\|_{\mathrm{L}_{2}(\Omega)}^{2}= & \int_{\Omega}(\nabla z-\nabla \widetilde{z}) \cdot\left(\nabla E^{\mathscr{R}}\left(u_{h}\right)-\nabla_{h} u_{h}\right) \mathrm{d} x \\
& \quad+\int_{\mathscr{E}}\left\{[\nabla z-\nabla \widetilde{z}\} \cdot \llbracket u_{h} \rrbracket \mathrm{d} s\right. \\
\leq & \left\|h^{-1}(\nabla z-\nabla \widetilde{z})\right\|_{\mathrm{L}_{2}(\Omega)}\left\|h\left(\nabla E^{\mathscr{R}}\left(u_{h}\right)-\nabla_{h} u_{h}\right)\right\|_{\mathrm{L}_{2}(\Omega)} \\
& \quad+\| h^{-1 / 2}\left\{[\nabla z-\nabla \widetilde{z}\}\left\|_{\mathrm{L}_{2}(\mathscr{E})}\right\| h^{1 / 2} \llbracket u_{h} \rrbracket \|_{\mathrm{L}_{2}(\mathscr{E})} .\right.
\end{aligned}
$$

The result follows using the approximability of the $\mathrm{HCT}(k+2)$ space [9] that can be inferred through the dimensional analysis in Definition 2.3 and the regularity of the dual problem, specifically

$$
\begin{aligned}
\left\|h^{-1}(\nabla z-\nabla \widetilde{z})\right\|_{\mathrm{L}_{2}(\Omega)}+\| h^{-1 / 2}\left\{\{\nabla z-\nabla \widetilde{z}\} \|_{\mathrm{L}_{2}(\mathscr{E})}\right. & \leq C|z|_{\mathrm{H}^{2}(\Omega)} \\
& \leq C\left\|E^{\mathscr{R}}\left(u_{h}\right)-u_{h}\right\|_{\mathrm{L}_{2}(\Omega)},
\end{aligned}
$$

thereby concluding the proof. 
Theorem 2.9 (inf-sup stability over $W(h)$ ) For polynomial degree $k \geq 2$ there exists $a \gamma_{h}>0$, depending on the quasiuniformity constant $C_{q u}$, such that, when $\sigma_{0}, \sigma_{1}$ are chosen large enough, we have for all $w_{h} \in \mathbb{V}$

$$
\sup _{\widetilde{v} \in W(h)} \frac{\mathscr{A}_{h}\left(w_{h}, \widetilde{v}\right)}{\|\widetilde{v}\|_{0, h}} \geq \gamma_{h}\left\|w_{h}\right\|_{2, h},
$$

where $W(h):=\mathbb{V}+H C T(k+2)$.

Proof The proof consists of two steps. We first show for a given $w_{h} \in \mathbb{V}$ that there exists a $\tilde{v} \in W(h)$ such that

$$
\mathscr{A}_{h}\left(w_{h}, \widetilde{v}\right) \geq C\left(\min _{x \in \Omega} h\right)^{2}\left\|w_{h}\right\|_{2, h}^{2}
$$

and that

$$
\|\widetilde{v}\|_{\mathrm{L}_{2}(\Omega)} \leq C\left(\max _{x \in \Omega} h\right)^{2}\left\|w_{h}\right\|_{2, h}
$$

which, along with the quasi-uniformity assumption on the mesh, yields the inf-sup condition (22).

Firstly note that, after an integration by parts, the IP method (7) can be written as

$$
\begin{aligned}
\mathscr{A}_{h}\left(w_{h}, v_{h}\right)= & \int_{\Omega}-\Delta_{h} w_{h} v_{h} \mathrm{~d} x+\int_{\mathscr{E}} \llbracket \nabla w_{h} \rrbracket\left\{v_{h}\right\} \mathrm{d} s-\int_{\mathscr{E} \cup \partial \Omega} \llbracket w_{h} \rrbracket\left\{\left\{\nabla v_{h}\right\}\right\} \mathrm{d} s \\
& +\int_{\mathscr{E} \cup \partial \Omega} \frac{\sigma_{0}}{h} \llbracket w_{h} \rrbracket \cdot \llbracket v_{h} \rrbracket \mathrm{d} s+\int_{\mathscr{E}} \sigma_{1} h \llbracket \nabla w_{h} \rrbracket \cdot \llbracket \nabla v_{h} \rrbracket \mathrm{d} s .
\end{aligned}
$$

Upon setting $\widetilde{v}=w_{h}-E^{\mathscr{R}}\left(w_{h}\right)-\alpha h^{2} \Delta_{h} w_{h}$, for some parameter $\alpha \in \mathbb{R}$ to be chosen below, we compute

$$
\begin{aligned}
\mathscr{A}_{h}\left(w_{h}, \widetilde{v}\right)= & \alpha\left\|h \Delta_{h} w_{h}\right\|_{\mathrm{L}_{2}(\Omega)}^{2}+\sigma_{1}\left\|h^{1 / 2} \llbracket \nabla w_{h} \rrbracket\right\|_{\mathrm{L}_{2}(\mathscr{E})}^{2}+\sigma_{0}\left\|h^{-1 / 2} \llbracket w_{h} \rrbracket\right\|_{\mathrm{L}_{2}(\mathscr{E} \cup \partial \Omega)}^{2} \\
& -\int_{\Omega} \Delta_{h} w_{h}\left(w_{h}-E^{\mathscr{R}}\left(w_{h}\right)\right) \mathrm{d} x \\
& +\int_{\mathscr{E}} \llbracket \nabla w_{h} \rrbracket\left\{\left\{w_{h}-E^{\mathscr{R}}\left(w_{h}\right)\right\} \mathrm{d} s-\int_{\mathscr{E} \cup \partial \Omega} \llbracket w_{h} \rrbracket \cdot\left\{\left\{\nabla\left(w_{h}-E^{\mathscr{R}}\left(w_{h}\right)\right)\right\}\right\} \mathrm{d} s\right. \\
& -\alpha \int_{\mathscr{E}} h^{2} \llbracket \nabla w_{h} \rrbracket\left\{\left\{w_{h}\right\}\right] \mathrm{d} s+\alpha \int_{\mathscr{E} \cup \partial \Omega} h^{2} \llbracket w_{h} \rrbracket \cdot\left\{\left[\nabla \Delta w_{h}\right\}\right\} \mathrm{d} s \\
& -\alpha \sigma_{1} \int_{\mathscr{E}} h^{3} \llbracket \nabla w_{h} \rrbracket \llbracket \nabla \Delta w_{h} \rrbracket \mathrm{d} s-\sigma_{0} \alpha \int_{\mathscr{E} \cup \partial \Omega} h \llbracket w_{h} \rrbracket \cdot \llbracket \Delta w_{h} \rrbracket \mathrm{d} s .
\end{aligned}
$$

The orthogonality property of the $\mathrm{HCT}(k+2)$-Ritz reconstruction (15) yields

$$
\int_{\Omega}-\Delta_{h} w_{h}\left(w_{h}-E^{\mathscr{R}}\left(w_{h}\right)\right) \mathrm{d} x=\int_{\Omega}\left(\Delta E^{\mathscr{R}}\left(w_{h}\right)-\Delta_{h} w_{h}\right)\left(w_{h}-E^{\mathscr{R}}\left(w_{h}\right)\right) \mathrm{d} x .
$$


Repeated use of the Cauchy-Schwarz inequality, therefore, gives

$$
\begin{gathered}
\mathscr{A}_{h}\left(w_{h}, \widetilde{v}\right) \geq \alpha\left\|h \Delta_{h} w_{h}\right\|_{\mathrm{L}_{2}(\Omega)}^{2}+\sigma_{1}\left\|h^{1 / 2} \llbracket \nabla w_{h} \rrbracket\right\|_{\mathrm{L}_{2}(\mathscr{E})}^{2}+\sigma_{0}\left\|h^{-1 / 2} \llbracket w_{h} \rrbracket\right\|_{\mathrm{L}_{2}(\mathscr{E} \cup \partial \Omega)}^{2} \\
-\left\|h\left(\Delta_{h} w_{h}-\Delta E^{\mathscr{R}}\left(w_{h}\right)\right)\right\|_{\mathrm{L}_{2}(\Omega)}\left\|h^{-1}\left(w_{h}-E^{\mathscr{R}}\left(w_{h}\right)\right)\right\|_{\mathrm{L}_{2}(\Omega)} \\
-\left\|h^{1 / 2} \llbracket \nabla w_{h} \rrbracket\right\|_{\mathrm{L}_{2}(\mathscr{E})}\left\|h^{-1 / 2} \llbracket\left\{w_{h}-E^{\mathscr{R}}\left(w_{h}\right)\right\}\right\|_{\mathrm{L}_{2}(\mathscr{E})} \\
-\left\|h^{-1 / 2} \llbracket w_{h} \rrbracket\right\|_{\mathrm{L}_{2}(\mathscr{E} \cup \partial \Omega)} \| h^{1 / 2}\left\{\left\{\nabla w_{h}-\nabla E^{\mathscr{R}}\left(w_{h}\right)\right\} \|_{\mathrm{L}_{2}(\mathscr{E} \cup \partial \Omega)}\right. \\
-\alpha\left\|h^{1 / 2} \llbracket \nabla w_{h} \rrbracket\right\|_{\mathrm{L}_{2}(\mathscr{E})}\left\|h^{3 / 2} \llbracket\left\{w_{h}\right\}\right\| \|_{\mathrm{L}_{2}(\mathscr{E})} \\
\left.-\alpha\left\|h^{-1 / 2} \llbracket w_{h} \rrbracket\right\|_{\mathrm{L}_{2}(\mathscr{E} \cup \partial \Omega)} \| h^{5 / 2} \llbracket \nabla \Delta w_{h}\right\} \|_{\mathrm{L}_{2}(\mathscr{E} \cup \partial \Omega)} \\
-\sigma_{1} \alpha\left\|h^{1 / 2} \llbracket \nabla w_{h} \rrbracket\right\|_{\mathrm{L}_{2}(\mathscr{E})}\left\|h^{5 / 2} \llbracket \nabla \Delta w_{h} \rrbracket\right\|_{\mathrm{L}_{2}(\mathscr{E})} \\
-\sigma_{0} \alpha\left\|h^{-1 / 2} \llbracket w_{h} \rrbracket\right\|_{\mathrm{L}_{2}(\mathscr{E} \cup \partial \Omega)}\left\|h^{3 / 2} \llbracket \Delta w_{h} \rrbracket\right\|_{\mathrm{L}_{2}(\mathscr{E} \cup \partial \Omega)} \\
=: \alpha\left\|h \Delta_{h} w_{h}\right\|_{\mathrm{L}_{2}(\Omega)}^{2}+\sigma_{1}\left\|h^{1 / 2} \llbracket \nabla w_{h} \rrbracket\right\|_{\mathrm{L}_{2}(\mathscr{E})}^{2} \\
+\sigma_{0}\left\|h^{-1 / 2} \llbracket w_{h} \rrbracket\right\|_{\mathrm{L}_{2}(\mathscr{E} \cup \partial \Omega)}^{2}-\sum_{i=1}^{7} \mathscr{I}_{i} .
\end{gathered}
$$

We proceed to bound each of the terms $\mathscr{I}_{i}$ individually. Note that in view of scaling and inverse inequalities we have for any $w_{h} \in \mathbb{V}$ :

$$
\begin{aligned}
\left\|\left\{\left\{w_{h}\right\}\right\}\right\|_{\mathrm{L}_{2}(e)} & \leq C_{1}\left\|h^{-1 / 2} w_{h}\right\|_{\mathrm{L}_{2}\left(\bar{K}_{1} \cup \bar{K}_{2}\right)} \\
\left\|\left\{\left\{\nabla w_{h}\right\}\right\}\right\|_{\mathrm{L}_{2}(e)} & \leq C_{2}\left\|h^{-3 / 2} w_{h}\right\|_{\mathrm{L}_{2}\left(\bar{K}_{1} \cup \bar{K}_{2}\right)}
\end{aligned}
$$

for any edge/face $e:=\bar{K}_{1} \cap \bar{K}_{2} \in \mathscr{E}$, and elements $K_{1}, K_{2} \in \mathscr{T}$, with $C_{1}, C_{2}$ depending only on the mesh-regularity and shape-regularity constants.

For $\mathscr{I}_{1}$, in view of Lemma 2.8 , we have

$$
\mathscr{I}_{1} \leq C_{3}\left(\left\|h^{1 / 2} \llbracket \nabla w_{h} \rrbracket\right\|_{\mathrm{L}_{2}(\mathscr{E})}^{2}+\left\|h^{-1 / 2} \llbracket w_{h} \rrbracket\right\|_{\mathrm{L}_{2}(\mathscr{E})}^{2}\right)
$$

with constant $C_{3}>0$ being the maximum of all constants in (16) for all $\alpha$.

For $\mathscr{I}_{2},(29)$ and Lemma 2.8 yield

$$
\begin{aligned}
\mathscr{I}_{2} & \leq C_{1} C_{3}^{1 / 2}\left\|h^{1 / 2} \llbracket \nabla w_{h} \rrbracket\right\|_{\mathrm{L}_{2}(\mathscr{E})}\left(\left\|h^{1 / 2} \llbracket \nabla w_{h} \rrbracket\right\|_{\mathrm{L}_{2}(\mathscr{E})}^{2}+\left\|h^{-1 / 2} \llbracket w_{h} \rrbracket\right\|_{\mathrm{L}_{2}(\mathscr{E})}^{2}\right)^{1 / 2} \\
& \leq C_{1}^{2} C_{3}\left\|h^{1 / 2} \llbracket \nabla w_{h} \rrbracket\right\|_{\mathrm{L}_{2}(\mathscr{E})}^{2}+\frac{C_{1}^{2} C_{3}}{2}\left\|h^{-1 / 2} \llbracket w_{h} \rrbracket\right\|_{\mathrm{L}_{2}(\mathscr{E})}^{2} .
\end{aligned}
$$

For $\mathscr{I}_{3},(30)$ and Lemma 2.8 yield

$$
\begin{aligned}
\mathscr{I}_{3} & \leq C_{2} C_{3}^{1 / 2}\left\|h^{-1 / 2} \llbracket w_{h} \rrbracket\right\|_{\mathrm{L}_{2}(\mathscr{E} \cup \partial \Omega)}\left(\left\|h^{1 / 2} \llbracket \nabla w_{h} \rrbracket\right\|_{\mathrm{L}_{2}(\mathscr{E})}^{2}+\left\|h^{-1 / 2} \llbracket w_{h} \rrbracket\right\|_{\mathrm{L}_{2}(\mathscr{E})}^{2}\right)^{1 / 2} \\
& \leq \frac{C_{2}^{2} C_{3}}{2}\left\|h^{1 / 2} \llbracket \nabla w_{h} \rrbracket\right\|_{\mathrm{L}_{2}(\mathscr{E} \cup \partial \Omega)}+C_{2}^{2} C_{3}\left\|h^{-1 / 2} \llbracket w_{h} \rrbracket\right\|_{\mathrm{L}_{2}(\mathscr{E})} .
\end{aligned}
$$


For $\mathscr{I}_{4}$, we have

$$
\begin{aligned}
\mathscr{I}_{4} \leq & C_{1} \alpha\left\|h^{1 / 2} \llbracket \nabla w_{h} \rrbracket\right\|_{\mathrm{L}_{2}(\mathscr{E})}\left\|h \Delta w_{h}\right\|_{\mathrm{L}_{2}(\Omega)} \leq \epsilon_{4} \alpha\left\|h \Delta w_{h}\right\|_{\mathrm{L}_{2}(\Omega)}^{2} \\
& +\frac{C_{1}^{2} \alpha}{4 \epsilon_{4}}\left\|h^{1 / 2} \llbracket \nabla w_{h} \rrbracket\right\|_{\mathrm{L}_{2}(\mathscr{E})}^{2},
\end{aligned}
$$

for any $\epsilon_{4}>0$, while for $\mathscr{I}_{5}$, we get

$$
\begin{aligned}
\mathscr{I}_{5} \leq & C_{2} \alpha\left\|h^{-1 / 2} \llbracket w_{h} \rrbracket\right\|_{\mathrm{L}_{2}(\mathscr{E} \cup \partial \Omega)}\left\|h \Delta w_{h}\right\|_{\mathrm{L}_{2}(\Omega)} \leq \epsilon_{5} \alpha\left\|h \Delta w_{h}\right\|_{\mathrm{L}_{2}(\Omega)}^{2} \\
& +\frac{C_{2}^{2} \alpha}{4 \epsilon_{5}}\left\|h^{-1 / 2} \llbracket w_{h} \rrbracket\right\|_{\mathrm{L}_{2}(\mathscr{E} \cup \partial \Omega)}^{2} .
\end{aligned}
$$

for any $\epsilon_{5}>0$; similarly for $\mathscr{I}_{6}$ and for any $\epsilon_{6}>0$, we have

$$
\begin{aligned}
\mathscr{I}_{6} \leq & C_{2} \sigma_{1} \alpha\left\|h^{1 / 2} \llbracket \nabla w_{h} \rrbracket\right\|_{\mathrm{L}_{2}(\mathscr{E})}\left\|h \Delta w_{h}\right\|_{\mathrm{L}_{2}(\Omega)} \leq \epsilon_{6} \alpha\left\|h \Delta w_{h}\right\|_{\mathrm{L}_{2}(\Omega)}^{2} \\
& +\frac{C_{2}^{2} \sigma_{1}^{2} \alpha}{4 \epsilon_{6}}\left\|h^{1 / 2} \llbracket \nabla w_{h} \rrbracket\right\|_{\mathrm{L}_{2}(\mathscr{E})}^{2} .
\end{aligned}
$$

Finally, the last term $\mathscr{I}_{7}$ can be bounded as follows:

$$
\begin{aligned}
\mathscr{I}_{7} \leq & C_{1} \sigma_{0} \alpha\left\|h^{-1 / 2} \llbracket w_{h} \rrbracket\right\|_{\mathrm{L}_{2}(\mathscr{E} \cup \partial \Omega)}\left\|h \Delta_{h} w_{h}\right\|_{\mathrm{L}_{2}(\Omega)} \leq \epsilon_{7} \alpha\left\|h \Delta_{h} w_{h}\right\|_{\mathrm{L}_{2}(\Omega)}^{2} \\
& +\frac{C_{1}^{2} \sigma_{0}^{2} \alpha}{4 \epsilon_{7}}\left\|h^{-1 / 2} \llbracket w_{h} \rrbracket\right\|_{\mathrm{L}_{2}(\mathscr{E} \cup \partial \Omega)}^{2},
\end{aligned}
$$

for any $\epsilon_{7}>0$.

Collecting the results (31)-(37) and substituting this into (28) we deduce

$$
\begin{aligned}
\mathscr{A}_{h}\left(w_{h}, \widetilde{v}\right) \geq & \left\|h \Delta_{h} w_{h}\right\|_{\mathrm{L}_{2}(\Omega)}^{2} \alpha\left(1-\epsilon_{4}-\epsilon_{5}-\epsilon_{6}-\epsilon_{7}\right) \\
& +\left\|h^{1 / 2} \llbracket \nabla w_{h} \rrbracket\right\|_{\mathrm{L}_{2}(\mathscr{E})}^{2}\left(\sigma_{1}-C_{3}-C_{1}^{2} C_{3}-\frac{C_{2}^{2} C_{3}^{2}}{2}-\frac{C_{1}^{2} \alpha}{4 \epsilon_{4}}-\frac{C_{2}^{2} \sigma_{1}^{2} \alpha}{4 \epsilon_{6}}\right) \\
& +\left\|h^{-1 / 2} \llbracket w_{h} \rrbracket\right\|_{\mathrm{L}_{2}(\mathscr{E} \cup \Omega)}^{2}\left(\sigma_{0}-C_{3}-\frac{C_{1}^{2} C_{3}}{2}-C_{2}^{2} C_{3}-\frac{C_{2}^{2} \alpha}{4 \epsilon_{5}}-\frac{C_{1}^{2} \sigma_{0}^{2} \alpha}{4 \epsilon_{7}}\right) .
\end{aligned}
$$

To arrive to (23), we can choose $\epsilon_{4}=\epsilon_{5}=\epsilon_{6}=\epsilon_{7}=\frac{1}{5}, \alpha=\left(\max \left(\sigma_{0}^{2}, \sigma_{1}^{2}\right)\right)^{-1}$ and $\sigma_{0}$ and $\sigma_{1}$ large enough.

For (24), we use Lemma 2.8 to see that

$$
\|\widetilde{v}\|_{\mathrm{L}_{2}(\Omega)} \leq\left\|w_{h}-E^{\mathscr{R}}\left(w_{h}\right)\right\|_{\mathrm{L}_{2}(\Omega)}+\left\|\alpha h^{2} \Delta_{h} w_{h}\right\|_{\mathrm{L}_{2}(\Omega)} \leq C\left\|h^{2} w_{h}\right\|_{2, h},
$$

which, together with the quasiuniformity of the meshes, completes the proof. 
Lemma 2.10 (Stability of the Ritz projection) Let $R: W(h) \rightarrow \mathbb{V}$ denote the $\mathscr{A}_{h}(\cdot, \cdot)$ orthogonal projector into $\mathbb{V}$. Then, for $\widetilde{w} \in W(h)$, there exists a $C>0$, independent of $h$ but possibly dependent on the quasiuniformity constant, $C_{q u}$, such that

$$
\|R \tilde{w}\|_{\mathrm{L}_{2}(\Omega)} \leq C\left(\max _{x \in \Omega} h\|\nabla \widetilde{w}\|_{\mathrm{L}_{2}(\Omega)}+\|\widetilde{w}\|_{\mathrm{L}_{2}(\Omega)}\right) \leq C\|\widetilde{w}\|_{\mathrm{L}_{2}(\Omega)} .
$$

Proof Let $\widetilde{g} \in W(h)$ be the solution to the discrete dual problem such that

$$
\mathscr{A}_{h}(\widetilde{v}, \widetilde{g})=\langle R \widetilde{w}, \widetilde{v}\rangle \quad \forall \widetilde{v} \in W(h)
$$

Note that this is well posed owing to coercivity as long as the penalty parameters are tuned to account for the fact that $W(h)$ contains piecewise polynomials over the subpartition two degrees higher than $\mathbb{V}$ itself. Then, we have

$$
\begin{aligned}
\|R \widetilde{w}\|_{\mathrm{L}_{2}(\Omega)}^{2} & =\langle R \tilde{w}-\widetilde{w}, R \widetilde{w}\rangle+\langle\widetilde{w}, R \widetilde{w}\rangle \\
& =\mathscr{A}_{h}(R \tilde{w}-\widetilde{w}, \widetilde{g})+\langle\widetilde{w}, R \widetilde{w}\rangle .
\end{aligned}
$$

Let $\Pi: \mathrm{H}^{1}(\Omega) \rightarrow \mathbb{V} \cap \mathrm{H}_{0}^{1}(\Omega)$ a suitable projection with optimal approximation properties. Then

$$
\begin{aligned}
\|R \tilde{w}\|_{\mathrm{L}_{2}(\Omega)}^{2} & =\mathscr{A}_{h}(R \tilde{w}-\widetilde{w}, \tilde{g}-\Pi \widetilde{g})+\langle\widetilde{w}, R \widetilde{w}\rangle \\
& \leq\|h(R \tilde{w}-\widetilde{w})\|_{1, h}\left\|h^{-1}(\widetilde{g}-\Pi \widetilde{g})\right\|_{1, h}+\|\widetilde{w}\|_{\mathrm{L}_{2}(\Omega)}\|R \widetilde{w}\|_{\mathrm{L}_{2}(\Omega)},
\end{aligned}
$$

through the continuity of $\mathscr{A}_{h}(\cdot, \cdot)$. From the optimal approximation properties of the projection/interpolant $\Pi$, we have

$$
\left\|h^{-1}(\widetilde{g}-\Pi \widetilde{g})\right\|_{1, h}^{2} \leq C_{q u} \tilde{C}\|\widetilde{g}\|_{2, h}^{2},
$$

and, using the discrete regularity of $\widetilde{g}$ induced by the inf-sup condition in Theorem 2.9

$$
\gamma_{h}\|\widetilde{g}\|_{2, h} \leq \sup _{\widetilde{v} \in W(h)} \frac{\mathscr{A}_{h}(\tilde{g}, \widetilde{v})}{\|\widetilde{v}\|_{0, h}}=\sup _{\widetilde{v} \in W(h)} \frac{\langle R \widetilde{w}, \widetilde{v}\rangle}{\|\widetilde{v}\|_{0, h}} \leq C\|R \widetilde{w}\|_{\mathrm{L}_{2}(\Omega)} .
$$

Hence we see that

$$
\begin{aligned}
\|R \tilde{w}\|_{\mathrm{L}_{2}(\Omega)}^{2} & \leq C\left(\|h(R \tilde{w}-\widetilde{w})\|_{1, h}\|R \tilde{w}\|_{\mathrm{L}_{2}(\Omega)}+\|\widetilde{w}\|_{\mathrm{L}_{2}(\Omega)}\|R \widetilde{w}\|_{\mathrm{L}_{2}(\Omega)}\right) \\
& \leq C\left(\|h \widetilde{w}\|_{1, h}\|R \tilde{w}\|_{\mathrm{L}_{2}(\Omega)}+\|\widetilde{w}\|_{\mathrm{L}_{2}(\Omega)}\|R \widetilde{w}\|_{\mathrm{L}_{2}(\Omega)}\right)
\end{aligned}
$$

in view of the quasi-best approximation in $\|\cdot\|_{1, h}$ from (10). The conclusion follows from standard inverse inequalities.

Theorem 2.11 (inf-sup stability over $\mathbb{V}$ ) For polynomial degree $k \geq 2$ there exists a $\gamma_{h}>0$, independent of $h$, but dependent on $C_{q u}$ such that, when $\sigma_{0}, \sigma_{1} \geq 1$ are chosen as in Theorem 2.9, we have for all $w_{h} \in \mathbb{V}$ 


$$
\sup _{v_{h} \in \mathbb{V}} \frac{\mathscr{A}_{h}\left(w_{h}, v_{h}\right)}{\left\|v_{h}\right\|_{2, h}} \geq \gamma_{h}\left\|w_{h}\right\|_{0, h}
$$

Proof To show (47) we fix $w_{h}$ and let $\Phi \in \mathbb{V}$ be the solution of the dual problem

$$
\mathscr{A}_{h}(\Psi, \Phi)=\int_{\Omega} w_{h} \Psi \mathrm{d} x \quad \forall \Psi \in \mathbb{V}
$$

Following the same arguments as in the proof of Theorem 2.9, it is clear that there exists a $C>0$ such that

$$
C h^{2}\|\Phi\|_{2, h}^{2} \leq \mathscr{A}_{h}(\Phi, \widetilde{v}),
$$

where $\widetilde{v}:=\Phi-E^{\mathscr{R}}(\Phi)-\alpha h^{2} \Delta_{h} \Phi$. Now it is clear that

$$
\|\widetilde{v}\|_{\mathrm{L}_{2}(\Omega)}\|\Phi\|_{2, h} \leq C h^{2}\|\Phi\|_{2, h}^{2} \leq C \mathscr{A}_{h}(\Phi, \widetilde{v}),
$$

and hence in view of Lemma 2.10 we have, with $R$ denoting the $\mathscr{A}_{h}$ orthogonal projector into $\mathbb{V}$, that

$$
\|R \widetilde{v}\|_{\mathrm{L}_{2}(\Omega)} \leq C \max _{x \in \Omega} h\left(\|\nabla \widetilde{v}\|_{\mathrm{L}_{2}(\Omega)}+\|\widetilde{v}\|_{\mathrm{L}_{2}(\Omega)}\right) \leq C\|\widetilde{v}\|_{\mathrm{L}_{2}(\Omega)}
$$

through inverse inequalities. Hence

$$
\|R \tilde{v}\|_{\mathrm{L}_{2}(\Omega)}\|\Phi\|_{2, h} \leq C\|\widetilde{v}\|_{\mathrm{L}_{2}(\Omega)}\|\Phi\|_{2, h}
$$

Now arguing as in the Proof of Theorem 2.9, and noting the constant will now depend on $C_{q u}$, we may show that

$$
\|\widetilde{v}\|_{\mathrm{L}_{2}(\Omega)} \leq C\left\|h^{2} \Phi\right\|_{2, h}
$$

Combining the previous two inequalities yields

$$
\|R \widetilde{v}\|_{\mathrm{L}_{2}(\Omega)}\|\Phi\|_{2, h} \leq C\|h \Phi\|_{2, h}^{2} \leq C \mathscr{A}_{h}(\Phi, \widetilde{v})=C \mathscr{A}_{h}(\Phi, R \widetilde{v})
$$

concluding the proof.

Corollary 2.12 (Convergence) Let $u$ solve (1) and $u_{h} \in \mathbb{V}$ be the interior penalty approximation from (7), then, under the assumptions of Theorem 2.11,

$$
\left\|u-u_{h}\right\|_{0, h} \leq\left(1+\frac{C_{B}}{\gamma_{h}}\right) \inf _{w_{h} \in \mathbb{V}}\left\|u-w_{h}\right\|_{0, h}+\frac{1}{\gamma_{h}} \sup _{v_{h} \in \mathbb{V}} \frac{\mathscr{A}_{h}\left(u_{h}-u, v_{h}\right)}{\left\|v_{h}\right\|_{2, h}},
$$

where $\gamma_{h}$ is the discrete inf-sup constant and $C_{B}$ is the continuity constant. If $u \in$ $\mathrm{H}^{k+1}(\Omega)$ for $k \geq 2$ the following a priori bound holds:

$$
\left\|u-u_{h}\right\|_{0, h}+\left\|h\left(u-u_{h}\right)\right\|_{1, h}+\left\|h^{2}\left(u-u_{h}\right)\right\|_{2, h} \leq C h^{k+1}|u|_{k+1} .
$$


Proof Using the inf-sup condition from Theorem 2.11 we see for all $w_{h} \in \mathbb{V}$

$$
\begin{aligned}
\gamma_{h}\left\|w_{h}-u_{h}\right\|_{0, h} & \leq \sup _{v_{h} \in \mathbb{V}} \frac{\mathscr{A}_{h}\left(w_{h}-u_{h}, v_{h}\right)}{\left\|v_{h}\right\|_{2, h}} \\
& \leq \sup _{v_{h} \in \mathbb{V}} \frac{\mathscr{A}_{h}\left(w_{h}-u, v_{h}\right)}{\left\|v_{h}\right\|_{2, h}}+\sup _{v_{h} \in \mathbb{V}} \frac{\mathscr{A}_{h}\left(u-u_{h}, v_{h}\right)}{\left\|v_{h}\right\|_{2, h}}
\end{aligned}
$$

Now using the natural continuity bound

$$
\mathscr{A}_{h}\left(u-w_{h}, v_{h}\right) \leq C_{B}\left\|u-w_{h}\right\|_{0, h}\left\|v_{h}\right\|_{2, h}
$$

we see

$$
\left\|w_{h}-u_{h}\right\|_{0, h} \leq \frac{C_{B}}{\gamma_{h}}\left\|u-w_{h}\right\|_{0, h}+\frac{1}{\gamma_{h}} \sup _{v_{h} \in \mathbb{V}} \frac{\mathscr{A}_{h}\left(u-u_{h}, v_{h}\right)}{\left\|v_{h}\right\|_{2, h}} .
$$

Hence, in view of the triangle inequality

$$
\left\|u-u_{h}\right\|_{0, h} \leq\left(1+\frac{C_{B}}{\gamma_{h}}\right)\left\|u-w_{h}\right\|_{0, h}+\frac{1}{\gamma_{h}} \sup _{v_{h} \in \mathbb{V}} \frac{\mathscr{A}_{h}\left(u-u_{h}, v_{h}\right)}{\left\|v_{h}\right\|_{2, h}} .
$$

The bound (55) follows since $w_{h}$ was arbitrary and (56) follows from the best approximation of $\mathbb{V}$.

\section{Applications to problems with rough data}

In this section we examine some problems of the form

$$
\begin{aligned}
-\Delta u & =f \text { in } \Omega \\
u & =0 \text { on } \partial \Omega
\end{aligned}
$$

where $f$ may be as rough as $\mathrm{H}^{-2}(\Omega) \backslash \mathrm{H}^{-1}(\Omega)$ and so, $u \in \mathrm{L}_{2}(\Omega) \backslash \mathrm{H}^{1}(\Omega)$. This means that the problem (75) cannot be characterised through a weak formulation, rather an ultra weak formulation, whereby we seek $u \in \mathrm{L}_{2}(\Omega)$ such that

$$
\int_{\Omega}-u \Delta v \mathrm{~d} x=\langle f \mid v\rangle_{\mathrm{H}^{-2}(\Omega) \times \mathrm{H}_{0}^{2}(\Omega)} \quad \forall v \in \mathrm{H}_{0}^{2}(\Omega),
$$

and the right hand side of (62) is understood as a duality pairing. In this setting standard tools pertaining to the analysis of Galerkin methods may not apply, for example the Aubin-Nitsche duality argument.

However, the stabilised IP method is still well defined and the inf-sup condition still holds. Indeed, we define the modified IP method: seek $u_{h} \in \mathbb{V}$ such that

$$
\mathscr{A}_{h}\left(u_{h}, v_{h}\right)=\left\langle f \mid E_{0}^{\mathscr{R}}\left(v_{h}\right)\right\rangle_{\mathrm{H}^{-2}(\Omega) \times \mathrm{H}_{0}^{2}(\Omega)} \quad \forall v_{h} \in \mathbb{V},
$$


where $E_{0}^{2}: \mathbb{V} \rightarrow H_{0}^{2}(\Omega)$ is the modification of $E^{2}$ recovering onto $H_{0}^{2}(\Omega)$ which is constructed by setting to zero all the degrees of freedom on $\partial \Omega$. We note that Lemma 2.5 still holds verbatim when $E^{2}$ is replaced by $E_{0}^{2}$. The nonstandard definition of the right-hand side allows us to make sense of extremely rough source terms [15] by interpreting the right-hand side a a duality pairing. Correspondingly, we also denote by $E_{0}^{\mathscr{R}}$ the recovery given by Definition 2.7 when $E^{2}$ is replaced by $E_{0}^{2}$. Also, we note that Lemma 2.8 holds for $E_{0}^{\mathscr{R}}$ also.

Since the inf-sup condition given in Theorem 2.11 is a condition only on the operator itself, the best approximation result given in Corollary 2.12 holds true. The only uncertainty with the bound is the behaviour of the inconsistency term. The control of this term is the main motivation in the nonstandard definition of the right hand side of (63).

Theorem 3.1 (quasi-optimal error control for problems with rough data) Let $u \in$ $\mathrm{L}_{2}(\Omega)$ solve (62) and $u_{h} \in \mathbb{V}$ be the approximation defined through (63), then

$$
\left\|u-u_{h}\right\|_{\mathrm{L}_{2}(\Omega)} \leq C \inf _{w_{h} \in \mathbb{V}}\left(\left\|u-w_{h}\right\|_{\mathrm{L}_{2}(\Omega)}+\left\|h^{3 / 2} \llbracket \nabla w_{h} \rrbracket\right\|_{\mathrm{L}_{2}(\mathscr{E})}+\left\|h^{1 / 2} \llbracket w_{h} \rrbracket\right\|_{\mathrm{L}_{2}(\mathscr{E})}\right) .
$$

Proof The proof takes some inspiration from that of [17], where inconsistency terms arise from the fact that the solution of an elliptic problem may only lie in $\mathrm{H}^{1}(\Omega)$, for which the operator $\mathscr{A}_{h}\left(u, v_{h}\right)$ may not be well defined. Here, the situation is more involved, since the solution $u \in \mathrm{L}_{2}(\Omega) \backslash \mathrm{H}^{1}(\Omega)$.

Using the inf-sup condition from Theorem 2.11 we have

$$
\gamma_{h}\left\|w_{h}-u_{h}\right\|_{0, h} \leq \sup _{v_{h} \in \mathbb{V}} \frac{\mathscr{A}_{h}\left(w_{h}-u_{h}, v_{h}\right)}{\left\|v_{h}\right\|_{2, h}}
$$

Now, by adding and subtracting appropriate terms and using (62) and (63), we see

$$
\begin{aligned}
\mathscr{A}_{h}\left(w_{h}-u_{h}, v_{h}\right)= & \mathscr{A}_{h}\left(w_{h}, E_{0}^{\mathscr{R}}\left(v_{h}\right)\right)+\int_{\Omega} u \Delta E_{0}^{\mathscr{R}}\left(v_{h}\right) \mathrm{d} x+\mathscr{A}_{h}\left(w_{h}, v_{h}-E_{0}^{\mathscr{R}}\left(v_{h}\right)\right) \\
= & \mathscr{A}_{h}\left(w_{h}, E_{0}^{\mathscr{R}}\left(v_{h}\right)\right)+\int_{\Omega} u \Delta E_{0}^{\mathscr{R}}\left(v_{h}\right) \mathrm{d} x \\
& +\mathscr{A}_{h}\left(w_{h}-E_{0}^{2}\left(v_{h}\right), v_{h}-E_{0}^{\mathscr{R}}\left(v_{h}\right)\right)
\end{aligned}
$$

by the orthogonality properties of $E_{0}^{\mathscr{R}}\left(w_{h}\right)$ given in (14). Now we may use that

$$
\begin{aligned}
\int_{\Omega} u \Delta E_{0}^{\mathscr{R}}\left(v_{h}\right) \mathrm{d} x+\mathscr{A}_{h}\left(w_{h}, E_{0}^{\mathscr{R}}\left(v_{h}\right)\right) & \leq C\left\|u-w_{h}\right\|_{\mathrm{L}_{2}(\Omega)}\left\|\Delta E_{0}^{\mathscr{R}}\left(v_{h}\right)\right\|_{\mathrm{L}_{2}(\Omega)} \\
& \leq C\left\|u-w_{h}\right\|_{\mathrm{L}_{2}(\Omega)}\left\|v_{h}\right\|_{2, h},
\end{aligned}
$$

through the stability of $E^{\mathscr{R}}\left(v_{h}\right)$. 
Finally, using the approximation properties of $E_{0}^{\mathscr{R}}(\cdot)$ and $E_{0}^{2}(\cdot)$ we see

$$
\begin{aligned}
& \mathscr{A}_{h}\left(w_{h}-E_{0}^{2}\left(w_{h}\right), v_{h}-E_{0}^{\mathscr{R}}\left(v_{h}\right)\right) \\
& \leq C\left\|w_{h}-E_{0}^{2}\left(w_{h}\right)\right\|_{1, h}\left\|v_{h}-E_{0}^{\mathscr{R}}\left(v_{h}\right)\right\|_{1, h} \\
& \leq C\left(\left\|h^{3 / 2} \llbracket \nabla w_{h} \rrbracket\right\|_{\mathrm{L}_{2}(\mathscr{E})}+\left\|h^{1 / 2} \llbracket w_{h} \rrbracket\right\|_{\mathrm{L}_{2}(\mathscr{E})}\right)\left\|v_{h}\right\|_{2, h} .
\end{aligned}
$$

Substituting (67) and (68) into (66) we have

$$
\begin{aligned}
& \mathscr{A}_{h}\left(w_{h}-u_{h}, v_{h}\right) \\
& \quad \leq C\left(\left\|u-w_{h}\right\|_{\mathrm{L}_{2}(\Omega)}+\left\|h^{3 / 2} \llbracket \nabla w_{h} \rrbracket\right\|_{\mathrm{L}_{2}(\mathscr{E})}+\left\|h^{1 / 2} \llbracket w_{h} \rrbracket\right\|_{\mathrm{L}_{2}(\mathscr{E})}\right)\left\|v_{h}\right\|_{2, h},
\end{aligned}
$$

and hence

$$
\begin{aligned}
\left\|u-u_{h}\right\|_{\mathrm{L}_{2}(\Omega)} & \leq\left\|u-w_{h}\right\|_{\mathrm{L}_{2}(\Omega)}+\left\|u_{h}-w_{h}\right\|_{\mathrm{L}_{2}(\Omega)} \\
& \leq\left\|u-w_{h}\right\|_{\mathrm{L}_{2}(\Omega)}+\left\|u_{h}-w_{h}\right\|_{0, h} \\
& \leq C\left(\left\|u-w_{h}\right\|_{\mathrm{L}_{2}(\Omega)}+\left\|h^{3 / 2} \llbracket \nabla w_{h} \rrbracket\right\|_{\mathrm{L}_{2}(\mathscr{E})}+\left\|h^{1 / 2} \llbracket w_{h} \rrbracket\right\|_{\mathrm{L}_{2}(\mathscr{E})}\right)
\end{aligned}
$$

as required.

\subsection{Numerical experiments}

The implementation of all the numerical experiments was performed in Matlab ${ }^{\circledR}$ on a laptop computer with a $2.3 \mathrm{GHz}$ Intel i7 processor and $16 \mathrm{~GB}$ of RAM. All computations took less than 2 min on this machine.

\subsubsection{Test 1: an one-dimensional example}

We begin by assessing the method (63) for $d=1$. We set $\Omega=(0,1)$ and consider the problem of finding $u$ such that

$$
\begin{aligned}
-u^{\prime \prime} & =\delta_{\bar{x}}^{\prime} \quad \text { in } \Omega \\
u=0 & \text { on } \partial \Omega,
\end{aligned}
$$

where $\delta_{\bar{x}}^{\prime}$ denotes the distributional derivative of the Dirac distribution at a point $\bar{x} \in \Omega$. This one-dimensional problem was a motivating example in the classical work of Babuška and Osborn [3]; this computation is included here as a tribute to that inspiring work.

For this problem we can even characterise a distributional solution, indeed we have that

$$
u(x)= \begin{cases}-x & \text { when } x<\bar{x} \\ 1-x & \text { when } x>\bar{x}\end{cases}
$$


solves (75). If we assume that $\bar{x}$ does not lie on the skeleton of the triangulation we can define our approximation as seeking $u_{h} \in \mathbb{V}$ such that

$$
\mathscr{A}_{h}\left(u_{h}, v_{h}\right)=E_{0}^{\mathscr{R}}\left(v_{h}\right)^{\prime}(\bar{x}) \quad \forall v_{h} \in \mathbb{V} \text {. }
$$

Using Theorem 3.1 we are able to show this approximation satisfies the a priori bound

$$
\left\|u-u_{h}\right\|_{\mathrm{L}_{2}(\Omega)} \leq C h^{1 / 2-\epsilon} \quad \forall \epsilon>0
$$

since $\delta_{\bar{x}} \in \mathrm{H}^{-s}(\Omega)$ for all $s>1 / 2$.

We fix $k=2$ and solve (73) over a sequence of uniform meshes in 1d with $h=$ $1 / 2,1 / 4, \ldots, 1 / 1024$. We take $\bar{x}=1 / 2+\sqrt{2} / 100000$ as to not align it with the nodes of the mesh. In Fig. 2 we show the numerical approximation over the finest mesh along with the experimental order of convergence.

\subsubsection{Test 2: $d=2$ with a Dirac source term at a point}

We now take $\Omega=B(0,1)$, the open ball of radius 1 centred at the origin, and consider the problem of finding $u$ such that

$$
\begin{aligned}
-\Delta u=\delta_{0} & \text { in } \Omega \\
u=0 & \text { on } \partial \Omega .
\end{aligned}
$$

The exact solution $u$ is the fundamental solution of Laplace's problem

$$
u(\mathbf{x})=-\frac{1}{2 \pi} \log (|\mathbf{x}|)
$$

for which we have $u \in \mathrm{L}_{2}(\Omega) \backslash \mathrm{H}^{1}(\Omega)$.

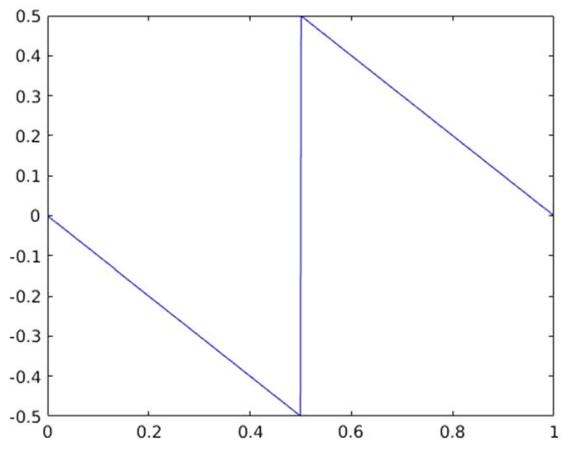

(a)

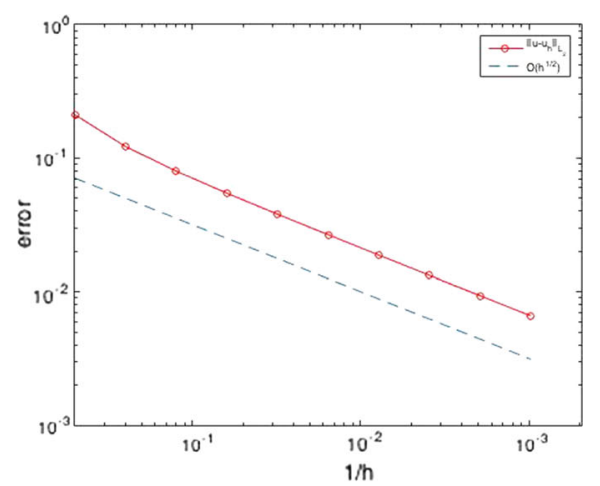

(b)

Fig. 2 In this experiment we test the $\mathrm{L}_{2}$ convergence of the interior penality method to approximate the distributional solution (72). Notice the error converges approximately like $\mathrm{O}\left(h^{1 / 2}\right)$. a The IP approximation. b Error and the experimental order of convergence 


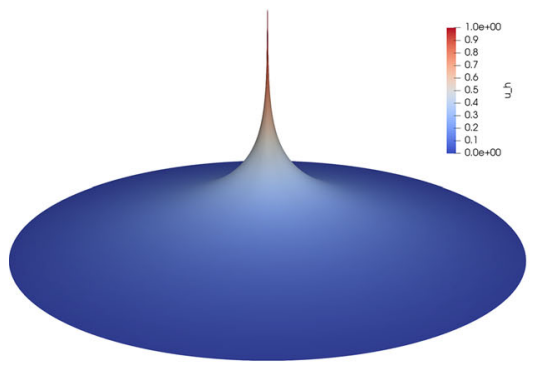

(a)

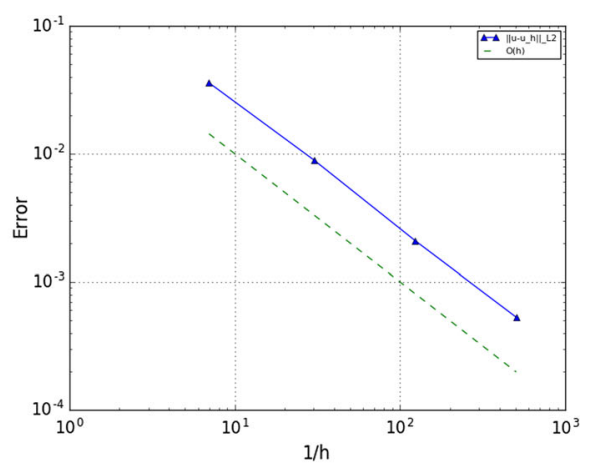

(b)

Fig. 3 We test the $\mathrm{L}_{2}$-norm convergence of the method (80) to approximate the fundamental solution of Laplace's problem (76). The error converges approximately like $\mathrm{O}(h)$. a The IP approximation. b Error and the experimental order of convergence

We define our approximation as seeking $u_{h} \in \mathbb{V}$ such that

$$
\mathscr{A}_{h}\left(u_{h}, v_{h}\right)=E_{0}^{\mathscr{R}}\left(v_{h}\right)(\bar{x}) \quad \forall v_{h} \in \mathbb{V},
$$

and, under the assumptions of Theorem 3.1, we are able to show that this approximation satifies the a priori bound

$$
\left\|u-u_{h}\right\|_{\mathrm{L}_{2}(\Omega)} \leq C h^{1-\epsilon} \quad \forall \epsilon>0
$$

This is in agreement with the results of [2] for conforming finite elements applied to this problem.

We fix $k=2$ and solve (80) over a sequence of unstructured, quasiuniform triangulations of $B(0,1)$. For the coarsest mesh we have $h \approx 0.13$ and for the most fine $h \approx 0.0019$. In Fig. 3, we show the numerical approximation over the finest mesh along with the error measured in the $\mathrm{L}_{2}$-norm and its convergence history. We remark, that the boundary approximation by straight-faced elements is not detrimental to the convergence rate in this case as the error is measured in the $\mathrm{L}_{2}$-norm.

\subsection{Test 3: $d=2$, rough source terms defined over an one-dimensional manifold}

We now test the proposed method on a more complicated problem, whereby, we set $\Omega=(0,1)^{2}$ and consider the problem of finding $u$ such that

$$
\begin{aligned}
-\Delta u & =\alpha \delta \mathscr{M}+(1-\alpha) \partial_{x} \delta_{\mathscr{M}} \quad \text { in } \Omega \\
u & =0 \quad \text { on } \partial \Omega,
\end{aligned}
$$

where $\alpha \in\{0,1\}$ and $\mathscr{M}:=\{(x, y):|x-1 / 2|<1 / 4$ and $y=1 / 2$ or $x=$ $1 / 2$ and $|y-1 / 2|<1 / 4\}$ is an one-dimensional manifold. When $\alpha=1$, we have $u \in \mathrm{H}^{1}(\Omega)$, whereas when $\alpha=0$, we have $u \in \mathrm{L}_{2}(\Omega) \backslash \mathrm{H}^{1}(\Omega)$. 


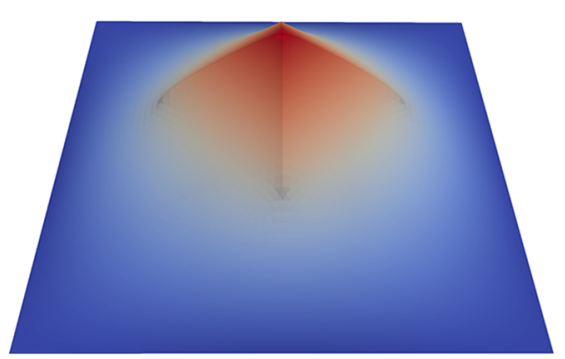

(a)

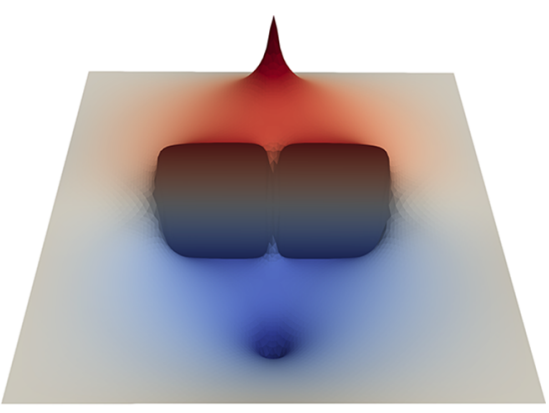

(b)

Fig. 4 The approximations to the solution of (79) produced by (80) for two different values of $\alpha$. The two dark regions on the right plot depict jump discontinuity. $\mathbf{a} \alpha=1$. $\mathbf{b} \alpha=0$

We seek $u_{h} \in \mathbb{V}$ such that

$$
\mathscr{A}_{h}\left(u_{h}, v_{h}\right)=\alpha E_{0}^{\mathscr{R}}\left(v_{h}\right)(\bar{x})+(1-\alpha) \partial_{x} E_{0}^{\mathscr{R}}\left(v_{h}\right)(\bar{x}) \quad \forall v_{h} \in \mathbb{V}, \bar{x} \in \mathscr{M}
$$

We fix $k=2$ and solve (80) over a uniform, criss-cross triangulation of $\Omega$ with $h \approx 0.015$. In Fig. 4 we show the numerical approximation over this mesh for both values of $\alpha$.

We conclude this exposition by noticing that, since the singularity in all tests were isolated, adaptive approximations should be able to recover best approximation. This motivates the extension of this analysis from the quite restrictive quasiuniform meshes to those that allow for some grading. Note the recent work [14] where, using a posteriori localisation techniques, analagous inf-sup results for the classical interior penalty $\mathrm{dG}$ scheme have been proven over meshes satisfying a mesh variation condition.

Acknowledgements TP acknowledges funding by the EPSRC grant EP/P000835/1.

Open Access This article is distributed under the terms of the Creative Commons Attribution 4.0 International License (http://creativecommons.org/licenses/by/4.0/), which permits unrestricted use, distribution, and reproduction in any medium, provided you give appropriate credit to the original author(s) and the source, provide a link to the Creative Commons license, and indicate if changes were made.

\section{References}

1. Arnold, D.N., Brezzi, F., Cockburn, B., Marini, L.D.: Unified analysis of discontinuous Galerkin methods for elliptic problems. SIAM J. Numer. Anal. 39(5), 1749-1779 (2001/02)

2. Babuška, I.: Error-bounds for finite element method. Numer. Math. 16(1), 322-333 (1971)

3. Babuška, I., Osborn, J.: Analysis of finite element methods for second order boundary value problems using mesh dependent norms. Numer. Math. 34(1), 41-62 (1980)

4. Babuška, I., Osborn, J., Pitkäranta, J.: Analysis of mixed methods using mesh dependent norms. Math. Comput. 35(152), 1039-1062 (1980)

5. Baker, G.A.: Finite element methods for elliptic equations using nonconforming elements. Math. Comput. 31(137), 45-59 (1977)

6. Brenner, S.C., Gudi, T., Sung, L.-Y.: A weakly over-penalized symmetric interior penalty method for the biharmonic problem. Electron. Trans. Numer. Anal. 37, 214-238 (2010) 
7. Brenner, S.C., Gudi, T., Sung, L.-Y.: An a posteriori error estimator for a quadratic $C^{0}$-interior penalty method for the biharmonic problem. IMA J. Numer. Anal. 30(3), 777-798 (2010)

8. Burman, E.: A unified analysis for conforming and nonconforming stabilized finite element methods using interior penalty. SIAM J. Numer. Anal. 43(5), 2012-2033 (2005). (electronic)

9. Ciarlet, P.G.: The Finite Element Method for Elliptic Problems. Studies in Mathematics and its Applications, vol. 4. North-Holland Publishing Co., Amsterdam (1978)

10. Douglas Jr., J., Dupont, T., Percell, P., Scott, R.: A family of $C^{1}$ finite elements with optimal approximation properties for various Galerkin methods for 2 nd and 4 th order problems. RAIRO Anal. Numér. 13(3), 227-255 (1979)

11. Ern, A., Guermond, J.-L.: Theory and Practice of Finite Elements, Volume of 159 Applied Mathematical Sciences. Springer, New York (2004)

12. Georgoulis, E.H., Hall, E., Makridakis, C.: A posteriori error control for discontinuous Galerkin methods for first order hyperbolic problems. In: Recent Developments in Discontinuous Galerkin Finite Element Methods for Partial Differential Equations: 2012 John H Barrett Memorial Lectures (2014)

13. Georgoulis, E.H., Houston, P., Virtanen, J.: An a posteriori error indicator for discontinuous Galerkin approximations of fourth-order elliptic problems. IMA J. Numer. Anal. 31(1), 281-298 (2011)

14. Georgoulis, E.H., Makridakis, C.G., Pryer, T.: Babuška-Osborn techniques in discontinuous Galerkin methods: $L^{2}$ error estimates for unstructured meshes. Submitted-Technical Report (2017). arXiV arXiv: 1704.05238

15. Georgoulis, E.H., Pryer, T.: Recovered finite element methods. Submitted-Technical Report (2017). ArXiV arXiv:1705.03649

16. Giesselmann, J., Makridakis, C., Pryer, T.: A posteriori analysis of discontinuous Galerkin schemes for systems of hyperbolic conservation laws. SIAM J. Numer. Anal. 53(3), 1280-1303 (2015)

17. Gudi, T.: A new error analysis for discontinuous finite element methods for linear elliptic problems. Math. Comput. 79(272), 2169-2189 (2010)

18. Karakashian, O.A., Pascal, F.: A posteriori error estimates for a discontinuous Galerkin approximation of second-order elliptic problems. SIAM J. Numer. Anal. 41(6), 2374-2399 (2003). (electronic)

19. Lai, Ming-Jun, Schumaker, Larry L.: Spline Functions on Triangulations, Volume of 110 Encyclopedia of Mathematics and its Applications. Cambridge University Press, Cambridge (2007)

20. Makridakis, C.G.: On the Babuška-Osborn approach to finite element analysis: $L^{2}$ estimates for unstructured meshes. Preprint (2016)

21. Nitsche, J.: Über ein Variationsprinzip zur Lösung von Dirichlet-Problemen bei Verwendung von Teilräumen, die keinen Randbedingungen unterworfen sind. Abh. Math. Sem. Univ. Hamburg 36, 9-15 (1971). (Collection of articles dedicated to Lothar Collatz on his sixtieth birthday)

22. Percell, P.: On cubic and quartic Clough-Tocher finite elements. SIAM J. Numer. Anal. 13(1), 100-103 (1976)

23. Pryer, T.: Discontinuous Galerkin methods for the $p$-biharmonic equation from a discrete variational perspective. Electron. Trans. Numer. Anal. 41, 328-349 (2014)

24. Pryer, T.: An a posteriori analysis of some inconsistent, nonconforming Galerkin methods approximating elliptic problems. Submitted-Technical Report (2015). ArXiV arXiv:1505.04318

25. Smears, I.: Nonoverlapping domain decomposition preconditioners for discontinuous Galerkin approximations of Hamilton-Jacobi-Bellman equations. J. Sci. Comput. 1-30 (2015) 